\title{
Commentary: Integrative Analysis of Multiple Sclerosis Using a Systems Biology Approach
}

\author{
Karla Cervantes Gracia', Holger Husi ${ }^{2,3 *}$
}

University of Monterrey, Health Sciences Division, Monterrey, 66238 , Mexico

${ }^{2}$ Institute of Cardiovascular and Medical Sciences, BHF Glasgow Cardiovascular Research Centre, University of Glasgow, Glasgow, G12 8TA, UK

${ }^{3}$ Department of Diabetes and Cardiovascular Science, University of the Highlands and Islands, Centre for Health Science, Inverness, IV2 3JH, UK

Article Info

\section{Article Notes}

Received: August 01, 2018

Accepted: September 12, 2018

\section{${ }^{*}$ Correspondence:}

Dr. Holger Husi, Institute of Cardiovascular and Medical Sciences, BHF Glasgow Cardiovascular Research Centre, University of Glasgow, Glasgow, G12 8TA, UK; E-mail: Holger.Husi@uhi.ac.uk.

C 2018 Husi H. This article is distributed under the terms of the Creative Commons Attribution 4.0 International License
Multiple sclerosis (MS) is considered the leading cause of nontraumatic disability worldwide in young adults with 2.3 million people estimated to live with MS in 2013, having a huge impact on their quality of life and economy ${ }^{1-3}$ MS is a chronic pro-inflammatory demyelinating disease of the central nervous system (CNS) that causes neurodegeneration. Its cause is uncertain, however, research suggests that MS is the result of a complex interplay between the immune system, environmental and genetic factors ${ }^{4-6}$. Despite more than 40 years of research, the lack of understanding and integration of key elements linked to MS, such as Epstein-Barr virus, Vitamin D, smoking, as well as genes associated with MS (i.e.. $H L A-D R B_{1}$ ), keeps its aetiology unknown ${ }^{7-10}$

Recent studies have focused on the identification of promising therapeutics, typically through 'hypothesis driven approaches. Haile and collaborators carried out a study regarding Rab32 role in MS, due to its regulatory activity over the mitochondria-associated membrane (MAM) and its relation with neuronal death. Their data showed in MS brain that endoplasmic reticulum stress induces Rab32, giving an insight of its role in MS pathology ${ }^{11}$. Another study focused on CD6, a protein previously linked with MS with an unknown function, and lack of in vivo studies with genetically engineered animals. Li et al. developed CD6 knockout mice to investigate the potential mechanisms in which CD6 is involved in MS. Additionally, by creating CD6 humanized-mice they evaluate CD6targeted reagents and tried to better understand its role ${ }^{12}$. Furthermore, Faissner et al. developed a systematic drug screening based on their previous studies about microglia inhibition, previously described as a pathological feature in all types of MS. In this study, they focused on other features known to be relevant in progressive-MS, such as disturbance of iron-mediated neurotoxicity, mitochondrial integrity and free radical scavenger. They investigated medications through cultures and experimentation in experimental autoimmune encephalomyelitis (EAE) models and found that several reduced T-lymphocytes proliferation and anti-oxidative potential ${ }^{13}$. The focus of these studies is based on what is previously known, therefore, they could benefit from an integrative approach by determining pathways affected and possible targets to investigate, leading to a more complete research and possibly more questions answered.

By following an integrative approach in the study titled "Integrative analysis of Multiple Sclerosis using a systems biology approach" we were able to pinpoint significant molecules and deregulated pathways that can give an insight of the underlying molecular mechanisms of MS. This 
study reports significant molecules present in peripheral blood of MS patients that can be considered as potential biomarkers by further and traditional validation.

Genomic large-scale datasets from previous MS studies were used to follow this approach. These datasets were correlated, and significant molecules and pathways for MS were determined. Several comparisons were made, depending on the characteristics of the samples from each study, and the comparisons were divided into groups since they were made between MS non-treaded and NonMS, as well as MS treated and MS Non-treated patients. These groups were analysed and the most significant ( $\mathrm{p}$-value $<0.05$ and $\log 2$ fold-changes $>1$ and $<-1$ ) and frequent molecules were determined. However, since the results from the Non-treated group were not significantly associated with gene ontologies nor pathways from the software used (ClueGo, Pathvisio), the pathways were a result of these molecules merged with the ones from the Treated group ${ }^{14,15}$. Having a bigger sample size, the Treated group represent most of the pathways highlighted through this study, which is one of its main weaknesses since they can be correlated with a response to MS treatment. However, this flaw was cleared when comparing our MS significant molecules with the ones also determined in this study from autoimmune disease datasets (SLE, RA, etc.). A complete overlap of the significant molecules between MS Treated and Autoimmune disease dataset groups were found, possibly meaning that these significant molecules can relate to a generic response in MS since it is unlikely and in some ways contradictory that MS pharmacological treatments result in a molecular phenotype mimicking autoimmune diseases ${ }^{16,17}$. These results could be interpreted as an MS generic response 'hidden' by a specific one obtained through the analysis of the Nontreated group.

Additionally, by comparing the statistically significant pattern of molecules found by these analyses, to EBVinfection peripheral blood datasets, no substantial correlation with MS was shown. The results obtained, represent indeed a specific molecular pattern to MS pathophysiology, where EBV can be a contributor but not the sole responsible of the molecular changes seen in MS patients' peripheral blood as previously established ${ }^{18}$.

One should bear in mind that besides a commonly followed approach to unravel MS aetiology, where a question is stated based on previous research and a hypothesis to be tested, a data-driven approach is based on the significance of the data, providing an insight of what is actually happening and of importance in a determined scenario ${ }^{19-21}$. This unbiased approach and analyses of a huge amount of data led to the rise of a new hypothesis that can be explored to better explain a disease $\mathrm{e}^{22}$. Both approaches have its qualities, however, some can prefer the hypothesis-driven one due to its certainty about the molecules and pathways they're focusing on. However, besides the many innovative omics-technologies that exist nowadays to develop this kind of approach, no considerable useful outputs have been established regarding MS aetiology. Furthermore, not all data-driven approaches result in significant correlations and hypotheses. GWAS and meta-analysis are examples in which a huge amount of data is handled and analysed resulting in significant molecules, but a lack of an in-depth data analysis and understanding of their correlation with the disease persists ${ }^{23-25}$.

The impact and clarity of results derived from a data driven-approach can sometimes be diminished due to its dependence on high-quality research, heterogeneity of the sample, sample size, as well reliable functional annotations, and the considerations taken when analyses are performed ${ }^{14,20,26}$. In this data-driven study this disadvantage was tackled by using the well curated PADB database (www.PADB.org). PADB database guarantees the use of appropriate curated resources by providing great coverage and record of different identifiers for each molecule from different databases and transcriptomic data platforms (Ensembl, UniProt, Affymetrix, Illumina, Agilent, etc.).

Despite the uncertainty that some researchers can establish about following data-driven approaches due to the functional annotations dependence and possibly misleading results, the methodology followed in this study reported molecules that have been previously studied and found to be involved in MS through other approaches ${ }^{27-29}$. Besides the new insights found regarding pathways and possible molecules that can be involved in MS, these findings backup the methodology and approach itself, supporting its implementation.

Research groups can benefit from this approach directly by relying on the statistical significance of their data. Moreover, once the data of interest is established, research groups with a significant amount of patients or samples at their disposition could follow a wet lab approach, obtaining reliable results and integrating data at different molecular levels, generating a better understanding of the disease or condition of interest.

A potential flaw of this study is its main focus on the analyses and integration of only transcriptomic and miRNA databases. The further integration of large-scale multi-omics datasets in the context of disease allows: an unbiased description of the disease as a whole, generation of novel hypotheses, and identification of potentially druggable targets. Therefore, in order to support, give more strength and complement the analyses done in this study, we propose these potential follow-ups. In this study, only peripheral blood samples were included, therefore a large-scale multi-omics analysis is suggested. Analysing 
multi-tissue and body-accessible fluid sources could give further confirmation that the molecules found in PBMC represent CNS events. These further studies would help define tissue-specific MS human molecular targets and fill gaps that could not be uncovered solely by this study due to the heterogeneity of the samples and other influencing factors. Also, by determining levels of specific molecular markers identified through this robust analysis, triggers of the different stages of MS can be determined, or elements that make the different stages of MS different from another over an established timeline.

Additionally, though MS doesn't quiet reflect the features to be classified as an autoimmune disorder, several articles suggest it is ${ }^{30-33}$. Furthermore, the MS autoimmune hallmark was "unmasked" by a treatment regime following this study approach. However, even though it was possible to identify some consistent molecules among studies that were previously associated with autoimmune aetiology and also new ones, as well as the identification of potential pathways associated with the disease, it is evident that other important molecules and elements exist in MS aetiology that could not be uncovered by this study due to heterogeneity and other influencing factors.

Therefore, in order to determine MS triggers of attack episodes and latent phases characteristic of autoimmune disorders, an MS treatment regime could be used to pursue future approaches. A longitudinal approach could be proposed, where MS patients are followed from the beginning of their treatments and at determined time points and compared with untreated MS patients as a control group to show the viral load, and give the "unmasking" feature this study showed. Levels of specific molecular markers, or even a whole screening at different levels (DNA, mRNA, protein) could be determined at different phases of the disease, in non-invasive samples (blood), and help us find an autoimmune baseline explanation for MS.

Furthermore, the creation of a Multiple Sclerosis (MuScle) database, part of the Pan-Omics Analysis Database (PADB) initiative, gives a significant impact directly associated with the data management done in this study, generating a complete curated and reliable MS database, giving the possibility of replication of this analysis and its usage in future MS research projects.

\section{References}

1. Multiple Sclerosis International Federation. "Atlas of MS 2013: mapping multiple sclerosis around the world." Mult Scler Int Fed. 2013: 1-28.

2. Thompson A.J., Baranzini S.E., Geurts J., et al. Multiple Sclerosis. The Lancet. 2018; 391(10130): 1622-1636.

3. Ernstsson O, Gyllensten H, Alexanderson K, et al. Cost of Illness of Multiple Sclerosis - A Systematic Review. PLOS ONE. 2016; 11(7): e0159129.

4. Tullman M.J. Overview of the epidemiology, diagnosis, and disease progression associated with multiple sclerosis. Am J Manag Care. 2013; 19: S15-20.

5. Pierrot-Deseilligny C, Souberbielle JC. Contribution of vitamin D insufficiency to the pathogenesis of multiple sclerosis. Ther Adv Neurol Disord. 2013; 6: 81-116.

6. Ascherio A, Munger KL. Epidemiology of multiple sclerosis: from risk factors to prevention. Semin Neurol. 2008; 28: 17-28.

7. Marashi SM, Shoja Z. "EBV Infection and Vitamin D in Multiple Sclerosis Patients." Nutrition and Lifestyle in Neurological Autoimmune Diseases. 2017: 9-20.

8. Saini V, Nadeem M, Kolb C, et al. "Vitamin D: Role in Pathogenesis of Multiple Sclerosis." Multiple Sclerosis. 2016: 127-152.

9. Bronson PG, Caillier S, Ramsay PP, et al. CIITA variation in the presence of HLA-DRB1* 1501 increases risk for multiple sclerosis. Hum Mol Genet. 2010; 19: 2331-40.

10. Marabita F, Almgren M, Sjöholm LK, et al. Smoking induces DNA methylation changes in Multiple Sclerosis patients with exposureresponse relationship. Sci Rep. 2017 Nov 6; 7(1): 14589.

11. Haile Y, Deng X, Ortiz-Sandoval C, et al. Rab32 connects ER stress to mitochondrial defects in multiple sclerosis. Journal of neuroinflammation. 2017; 14(1): 19.

12. Li Y, Singer NG, Whitbred J, et al. CD6 as a potential target for treating multiple sclerosis. Proc Natl Acad Sci USA. 2017; 114:2687-2692.

13. Faissner S, Mishra M, KaushikDK, et al. Systematic screening of generic drugs for progressive multiple sclerosis identifies clomipramine as a promising therapeutic. Nat Commun. 2017; 8(1), 1990.

14. Bindea G, Mlecnik B, Hackl H, et al. ClueGO: a Cytoscape plug-in to decipher functionally grouped gene ontology and pathway annotation networks. Bioinformatics. 2009; 25(8): 1091-1093.

15. Kutmon M, van Iersel MP, Bohler A, et al. PathVisio 3: An Extendable Pathway Analysis Toolbox. PLoS Comput Biol. 2015; 11(2): e1004085.

16. Javed A, Reder AT. Therapeutic role of beta-interferons in multiple sclerosis. Pharmacol Ther. 2006; 110(1): 35-56.

17. Hecker M, Hartmann C, Kandulski O, et al. Interferon-beta therapy in multiple sclerosis: the short-term and long-term effects on the patients' individual gene expression in peripheral blood. Mol Neurobiol (2013) 48: 737-56.

18. Fernández-Menéndez S, Fernández-Morán $M$, Fernández-Vega I, et al. Epstein-Barr virus and multiple sclerosis. From evidence to therapeutic strategies. J. Neurol. Sci. 2016; 361: 213-219.

19. Previdelli ÁN, de Andrade SC, Fisberg RM, et al. Using Two Different Approaches to Assess Dietary Patterns: Hypothesis-Driven and DataDriven Analysis. Nutrients. 2016; 8(10): 593.

20. He M, Liu P, Lawrence-Dill C. J. A hypothesis-driven approach to assessing significance of differences in RNA expression levels among specific groups of genes. Curr Plant Biology. 2017; 11: 46-51.

21. Oprea TI, Bologa CG, Brunak S, et al. Unexplored therapeutic opportunities in the human genome. Nat Rev Drug Discov. 2018; 17(5): 317-332.

22. Tan H, Chen R, Li W, et al. A systems biology approach to studying the molecular mechanisms of osteoblastic differentiation under cytokine combination treatment. NPJ Regen Med. 2017; 2: 5.

23. Hoffman JEI. Biostatistics for medical and biomedical practitioners: Meta-analysis. United States of America: Elsevier; 2015.

24. Gurevitch, J, Koricheva J, Nakagawa S, et al. Meta-analysis and the science of research synthesis. Nature. 2018; 555(7695):175-182.

25. Hounkpe BW, Fiusa MM, Colella MP, et al. Role of innate immunitytriggered pathways in the pathogenesis of Sickle Cell Disease: a metaanalysis of gene expression studies. Sci Rep. 2015; 5: 17822. 


\section{4-7}

26. Rhee SY, Wood V, Dolinski K, et al. Use and misuse of the gene ontology annotations. Nat Rev Genet. 2008; 9(7): 509-15.

27. Basivireddy J, Somvanshi RK, Romero IA, et al. Somatostatin preserved blood brain barrier against cytokine induced alterations: possible role in multiple sclerosis. Biochem Pharmacol. 2013; 86: 497-507.

28. Huss DJ, Winger RC, Cox GM, et al. TGF-beta signaling via Smad4 drives IL-10 production in effector Th1 cells and reduces T-cell tra cking in EAE. Eur J Immunol. 2011; 41:2987-96.

29. Waubant E, Gee L, Bacchetti P, et al. Relationship between serum levels of IL-10, MRI activity and interferon beta-1a therapy in patients with relapsing remitting MS. J Neuroimmunol. 2001; 112: 139-45.

30. Rodriguez M, Warrington AE, Pease LR. Invited article: human natural autoantibodies in the treatment of neurologic disease. Neurology. 2009;72(14): 1269-76.

31. Kennel De March A, De Bouwerie M, Kolopp-Sarda MN, et al. Antimyelin oligodendrocyte glycoprotein B-cell responses in multiple sclerosis. J Neuroimmunol. 2003; 135(1-2): 117-25.

32. Khalil M, Reindl M, Lutterotti A, et al. Epitope specificity of serum antibodies directed against the extracellular domain of myelin oligodendrocyte glycoprotein: influence of relapses and immunomodulatory treatments. J Neuroimmunol. 2006; 174(1-2): 147-56.

33. Steinman L. Immunology of relapse and remission in multiple sclerosis. Annu Rev Immunol. 2014; 32: 257-81. 